Article

\title{
Comparison of overground and resting endoscopy in the diagnosis of the simple and complex dynamic upper respiratory tract abnormalities in racehorses
}

\author{
Cihan KUMAS ${ }^{1}$, Mehmet MADEN ${ }^{2}$ and Orhan PINAR ${ }^{3 *}$
}

1 Affiliation 1; cihankumas@hotmail.com

2 Affiliation 2; mmaden@selcuk.edu.tr

3 Affiliation 3; vetorhanpinar@gmail.com

* Correspondence: vetorhanpinar@gmail.com ; Tel.:+905324543451

Simple Summary: It is so valuable to make a definitive diagnosis for finding the straightway of treatments of human and animal health for their welfare. In Equine Medicine respiratory problems usually occurs, after the orthopedic one, in a second position. The respiratory problems are derived from the horse owners, trainers, and jockeys as poor performance, unable to accelerate to speed at the end of the race and occurs abnormal respiratory noises. They come to the hospital for consulting their complaints, which are examined by vets by using a resting endoscopy. However, complaints are associated with exercise, examination to be done at rest, is not enough to make a definitive diagnosis. The aim of this study was to evaluate the source of their complaints, which were examined by using overground endoscopy during performing exercise in a natural field. The problems which are associated with the upper airway were seen clearly by dynamic endoscopy and determined that some of the cases occurred two or three disorders together, which can be changed the way of treatment. Results of the study showed the endoscopic findings to differ between resting and overground endoscopic examination and overground endoscopy could useful for an effective diagnosis and deciding treatment options.

\begin{abstract}
The purpose of the study was to find the source of complaints and to diagnose dynamic upper respiratory tract problems, which were derived from the horse owners, trainers, and jockeys, and to evaluate the overground endoscopic examination efficiency to determine the subclinical upper respiratory tract (URT) abnormalities, which were unable to diagnose with resting endoscopy. In the study, overground endoscopy has been used which nowadays there is a more useful and safe technique of performing endoscopy during ridden exercise in the natural field to diagnose dynamic upper respiratory tract (URT) abnormalities in comparison with the more traditional method of resting endoscopy. This study focused on 25 racehorses (Thoroughbred, n:22 and Arabian n: 3), which were admitted to Racehorse Hospital with complaints of fade abruptly and/or abnormal respiratory noise during the last meters of the race. All horses were examined for the complaints to be associated with systemic disorders and/or lameness, the racehorses with lameness and/or systemic disorders findings were excluded. Resting (post-exercise) and overground endoscopy by using Dr. Fritz ${ }^{\circledR}$ ETL-Equine Overground Endoscopy was performed on a polytrack racecourse in all racehorses. Observed abnormalities were recorded as simple and complex cases and the results of resting and dynamic examination were compared. It was concluded the overground endoscopic examination has a useful diagnostic value in determining the cause of abnormal respiratory noise and/or fade abruptly.
\end{abstract}

Keywords: Fade abruptly, abnormal respiratory noise, dynamic overground endoscopy, upper respiratory tract, horse.

\section{Introduction}


Dynamic upper respiratory tract (URT) obstruction occurs when soft tissue structure, nasopharynx and larynx, an anatomic or functional deformities and collapse as a result of negative airflow-inspire- pressure, which is a routine cause of abnormal airway noise and poor performance in equine athletes [1]. Negative pressure takes place in the Lower Respiratory Tract (LRT) and Upper Respiratory Tract (URT) when the diaphragm contracts throughout the inspiration. The significant increase in airflow volume during the exercise leads to a higher negative pressure. The nasopharynx and the larynx tend to collapse, because neither of them has a bony and/or stiff structure, and therefore active muscle work is needed to prevent this [2]. Vortices causing abnormal respiratory noise may be developed due to the obstruction of the respiratory tract [3]. Abnormal noises are classified by whistling/roaring, rasping, choking and rough/gargling. Whistling is usually heard when LLH grade III-IV diagnosed and rough is specific noise for the abnormalities of vocal fold collapse (VFC). These changes can only be seen during exercise and endoscopy of the upper respiratory tract is the best choice of estimating a definitive diagnosis in the horse with abnormal respiratory noise and poor performance. The upper airway is usually evaluated in horses by the use of resting or dynamic endoscopy and the retrospective studies have documented performing an endoscopy at rest can fail to discover abnormalities that occurs only under dynamic conditions [4]. Because of that dynamic endoscopic examination is regarded as the "gold standard" method for establishing a definitive diagnosis of URT obstruction [5-7].

Upper respiratory tract disorders of palatal dysfunction, which are Palatal instability (PI), dorsal displacement of the soft palate (DDSP), Laryngeal dysfunction, which are left laryngeal hemiplegia (LLH), vocal fold collapse (VFC), ventromedial luxation of the apex of the corniculate process (VLAC), and others, which are medial deviation of aryepiglottic folds (MDAF) and nasopharyngeal collapse (NPC), epiglottic entrapment (EE), and rostral displacement of the palatopharyngeal arch (RDPA) are considered to develop poor performance and abnormal noise [8,9]. Regarding the incidence in horses, it was stated that DDSP was seen highest (40\%), followed by RLN (20\%) [10]. Other effects of obstruction include NPC, VFC, MDAF, tracheal collapse, epiglottic entrapment and, VLAC [11].

There are two techniques of dynamic endoscopic examination, High-Speed Treadmill Examination (HSTE) and Overground (mobile) endoscopy (OE), which provides an alternative to HSTE for diagnosing URT obstruction in exercising horse, was founded for use in Equine Medicine in 2008 [12,13] and has been reported that to be safe and effective to visuals diagnostic dynamic images of the URT [14]. Overground endoscopy may be chosen instead of HSTE because it is more closely real competition [15].

Many studies to perform endoscopy during treadmill exercise have been established in the last two decades [8,9,16-19], however, performing a treadmill endoscopy in an artificial condition and is not representative of racing conditions, which are the presence of full tack, track surface, the weight of the rider (except the weight of equipment of OE $14 \mathrm{~kg}$ ), presence of sulky, some differences in head position, actions of riders [13,21].

In addition, HSTE technique, it has not been found it useful by horse owner and trainers because of the expense of equipment, personnel required, and time taken, and some horse injuries have been reported during the performing of HSTE [20].

Nowadays, the advance of overground endoscopy (OE) has enabled the monitoring of upper airway behavior with the recent development of a commercially available telemetric endoscope and a recording unit in normal exercising conditions, during ridden exercise in the natural field $[22,23]$.

The aim of this study was to investigate the source of complaints of fade abruptly and/or abnormal noise, which is derived from the horse owner, trainer, and jockey, come at the last meters were at full force by resting and overground endoscopy. 


\section{Materials and Methods}

This project was approved by the University of Selcuk, School of Veterinary Medicine, ethics and welfare committee. Informed consent to examine the horses was obtained from the trainer prior to the start of the project. If abnormalities were identified, advice on management and treatment options was given.

This study focused on 25 racehorses (Thoroughbred, n:22 and Arabian n: 3), which were admitted to the Racehorse Hospital with complaints of fade abruptly (FA) by trainers and/or abnormal respiratory noise during the last meters of the race, where to become evident which upper respiratory tract (URT) disorders are causing the poor performance and/or abnormal respiratory sound were reported by jockeys after the race. All horses were evaluated to associate any systemic disorders and lameness. Blood samples were analyzed and reported no systemic disorders findings. FA is an expression used by trainers and jockeys to describe when a horse slows down instead of going full force at the last meters of the race. If the horse can't increase its speed and suddenly slows down, then it referred to as fade abruptly.

Resting endoscopic examinations were done post-exercise which was immediately after the exercising tests in 30 minutes on 25 racehorses the morphology and function of the larynx and pharynx were evaluated. Overground endoscopy was performed in the natural race conditions and on a polytrack racecourse for the investigation of abnormal respiratory noise and/or fade abruptly by using Dr. Fritz ${ }^{\circledR}$ ETL-Equine Overground Endoscope which has a saddle, recording unit, battery, endoscope and snaffle with canelike apparatus.

The saddle is specifically designed for racehorses. The saddle has two cases, on the right and left sides. The endoscope is positioned on top of the saddle, then attached to the horse's mane and extended to the forehead section from between its two ears, where placed on the snaffle with the help of a cane like apparatus. Afterward, the endoscopy unit was placed from the right nostril and the distal tip was positioned within the nasopharynx at the level of the opening of the auditory tube diverticula, which allowed a clear view of the larynx as well as the caudal portion of the nasopharynx and soft palate and no sedatives were used. The total weight of this system is about $14 \mathrm{~kg}$. It was heavy equipment and unbale to remote by GPRS and flushing in case of collecting mucus in front of the camera during exercise. Because of these disadvantages, some of them were repeated until getting a clear one. Now slight one and remote control have already existed.

Recording started before the horses started to train, after preparation the horse was taken to the racecourse. The horses were ridden by their usual riders, who were trained as usual during OE. Horses were ridden first 800 meters were at canter then they galloped over 1000 meters and the last 600 meters were at full force on an $1870 \mathrm{~m}$ long polytrack, where the complaints of fade abruptly and/or abnormal noise coming from the last meters, when the functional change realized according to the decline of the horse performance and abnormal noise became to hear clearly. URT abnormalities based on recordings were documented.

\section{Results}

No obstructive URT disorders diagnosed neither resting nor overground endoscopic examination in one horse which showed only unknown abnormal noise wasn't definitive diagnosed. In this study, URT abnormalities were diagnosed by OE examination based on former studies by OE examination on the basis of former studies [6,19,24-26].

3.1. Findings Resting Endoscopy: 


\subsubsection{Palatal Dysfunction}

- Palatal Instability is progressive dorso-ventral billowing of the soft palate [19].[Figure 1]

\subsubsection{Laryngeal Dysfunction}

- The abnormalities diagnosed with the resting endoscopy were left laryngeal hemiplagea (LLH) which has 4 grade and to be exposed as Grade I, Grade II, Grade III and Grade IV which are classified according to movement of the left arytenoid cartilage and Grade I-II can rarely be diagnosed with resting endoscopy [4]. [Figure 2]

In resting endoscopy, LLH (n:8) which were Grade III and IV [Figure 1] and PI (n:6) abnormalities in 14 (58\%) of the 24 horses. However, it couldn't be diagnosed any abnormalities in 10 of the 24 horses while at resting endoscopy. [Table-1].

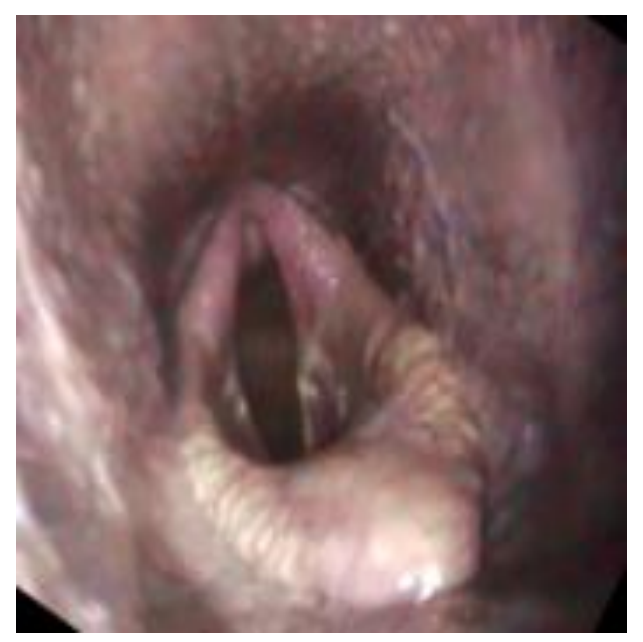

Figure 1. Palatal Instability (PI) to be diagnosed with resting endoscopy.

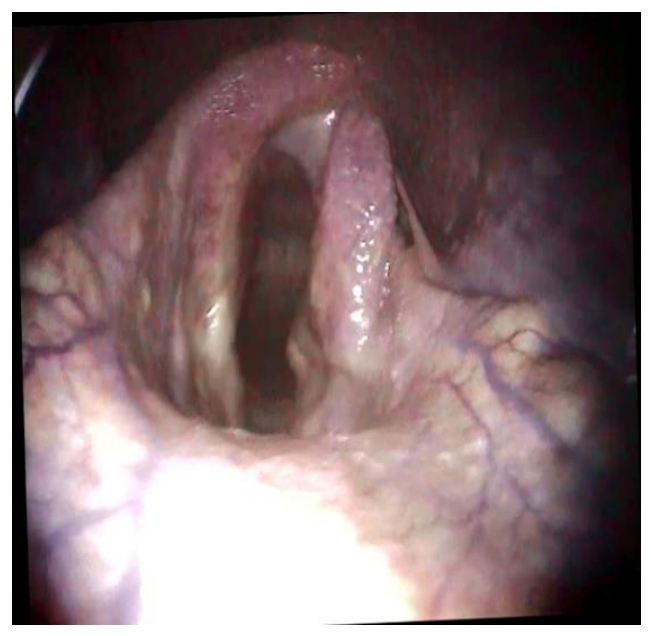

Figure 2. .Left Laryngeal Hemiplegia (LLH) Grade $I V$ to be diagnosed with resting endoscopy
Resting Endoscopic

\begin{tabular}{|c|c|c|}
\hline No & Noise & Noise Profile \\
\hline 1 & + & Whistling/Roaring \\
\hline 2 & - & - \\
\hline 3 & + & Unkonwn \\
\hline 4 & + & Loud \\
\hline 5 & - & - \\
\hline 6 & + & Rasping \\
\hline 7 & + & Whistling/Roaring \\
\hline 8 & + & Rough/Gurgling \\
\hline 9 & + & Loud \\
\hline 10 & - & - \\
\hline
\end{tabular}

Findings

LLH

$-$

$-$

PI

PI

PI

PI

LLH

PI
Overground Endoscopic Findings

LLH + MDAF + PI
NPC
-
VLAC + MDAF
DDSP
VLAC + MDAF + PI
MDAF + PI
DDSP
LLH + MDAF + DDSP
PI




$\begin{array}{lccccc}11 & - & + & \text { PI } & \text { PI } \\ 12 & + & \text { Whistling/Roaring } & + & \text { LLH } & \text { LLH + VFC + MDAF } \\ 13 & - & - & + & - & \text { NPC } \\ 14 & + & \text { Whistling/Roaring } & + & \text { LLH } & \text { LLH + VFC + PI } \\ 15 & + & \text { Whistling/Roaring } & + & \text { LLH } & \text { LLH + VFC + PI } \\ 16 & + & \text { Choking } & - & - & \text { VFC } \\ 17 & - & - & + & \text { PI } & \text { PI } \\ 18 & + & \text { Loud } & + & - & \text { MDAF + PI } \\ 19 & - & - & + & - & \text { NPC } \\ 20 & + & \text { Choking } & + & \text { LLH } & \text { LLH + PI } \\ 21 & + & \text { Unknown } & - & - & \text { PI } \\ 22 & - & - & + & - & \text { NPC } \\ 23 & + & \text { Loud } & + & \text { LLH } & \text { MDAF } \\ 24 & + & \text { Whistling/Roaring } & + & \text { LLH } & \text { LLH + VFC }\end{array}$

Table 1. This table shows the noise profile, are classified by horse owner, trainers and jockeys reported noise history, and comprehensions of 2 endoscopic findings on 25 racehorses. Left laryngeal hemiplegia (LLH), Palatal Instability (PI), Dorsal Displacement of Soft Palate (DDSP), Medial Deviation of Aryepiglottic Fold (MDAF), Nasopharyngeal Collapse (NPC), Vocal Fold Collapse (VFC), Ventro-medial Luxation of the Apex of the Corniculate Process (VLAC)

3.2. Findings Dynamic Respiratory Endoscopy:

3.2.1. Palatal Dysfunction

- Dorsal displacement of the soft palate (DDSP) is displacement of soft palate to the upper side of the epiglottis [17]. [Figure 3]

3.2.2. Laryngeal Dysfunction

- Vocal fold collapse (VFC) which is dynamic collapse of one or both vocal fold [19]. [Figure 4]

- Ventro-medial luxation of the apex of the corniculate process of the arytenoid (VLAC) is the apex left corniculate process ventrally under the right one [25].

3.2.3. Others

- Nasopharyngeal collapse (NPC) when dorsal or lateral walls of the pharynx come closer and narrowing the upper respiratory tract [26]. [Figure 5]

- Medial deviation of the aryepiglottic fold (MDAF) is medial displacement of the vertical margins of the aryepiglottic folds [24]. 


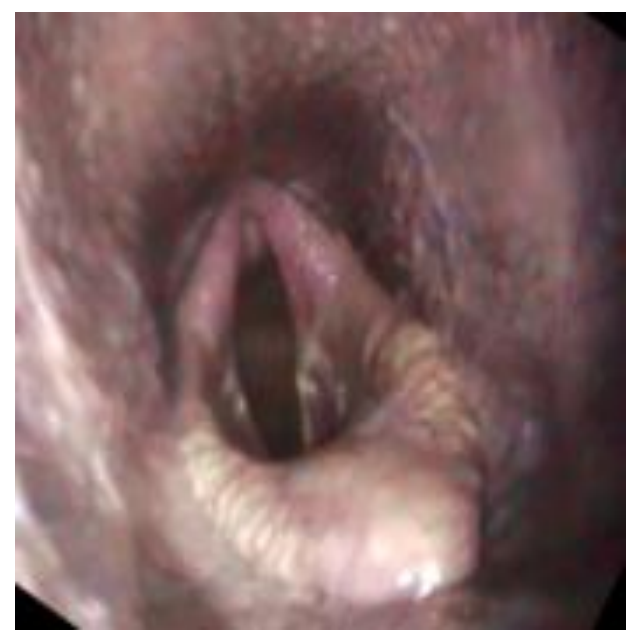

(a)

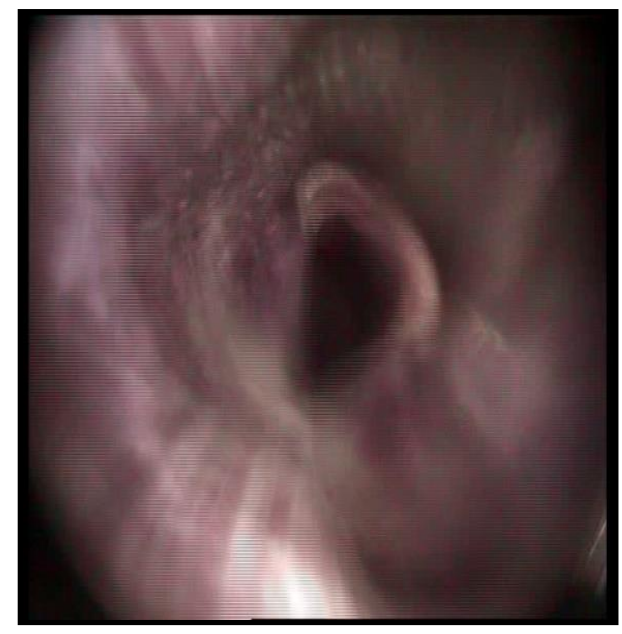

(b)

Figure 3. This figure compare the 2 endoscopic findings, (a) which shows Palatal Instability (PI) while at rest and (b) the same horse shows the Dorsal displacement of the soft palate (DDSP) during exercise.

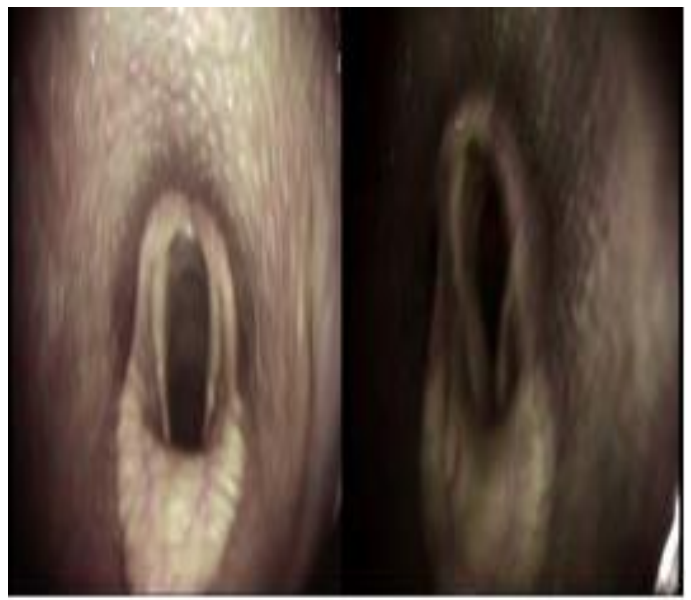

(a) (b)

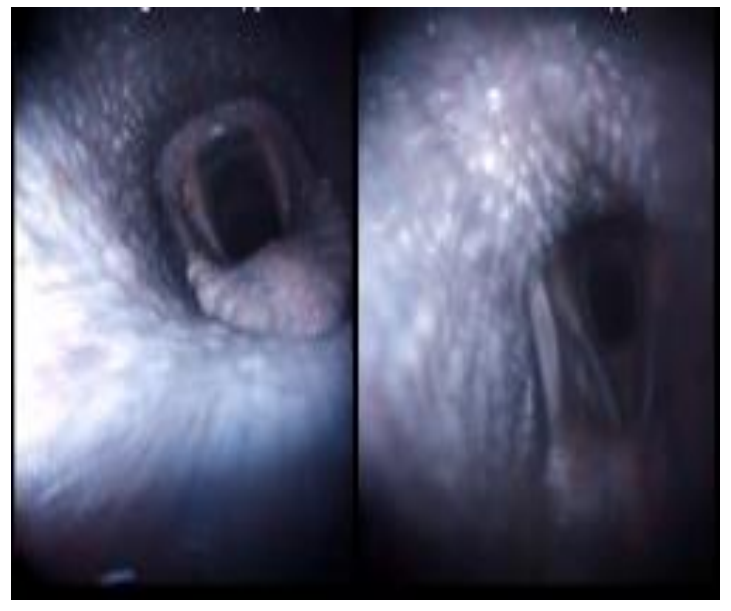

(a) (b)

Figure 4. This figure compare the 2 endoscopic findings (a), which shows left laryngeal hemiplagea (LLH) Grade III at rest and (b), shows LLH with Vocal Fold Collapse (VFC) during Figure 5. This figure shows (a) normal anatomy of URT, however the naso phrangeal collapse (NPC) could be evaluated (b) only during the exercise with overground endoscopy exercise as a complex case.

In all horses were seen well tolerated the equipment of overground endoscopy (OE) despite minor nasal discharge and failure of the flushing mechanism. NPC was diagnosed in 3 horses, DDSP in 2 horses, MDAF on 1 horse, and VFC on 1 horse in OE. Complex cases together with two and/or three abnormalities which were 11 different abnormalities determined totally, were diagnosed by OE. In the comparative evaluation of resting and $\mathrm{OE}$ examination, the diagnosis of complex cases was determined only with OE examination (Table-2). 
PI

DDSP

Laryngeal Dysfunction (Simple)

LLH

VFC

VLAC

Other (Simple)

MDAF

NPC

Complex Cases

$\begin{array}{ccr}\text { LLH + MDAF + PI } & 0 & 1 \\ \text { VLACP + MDAF } & 0 & 1 \\ \text { VLACP + MDAF + PI } & 0 & 1 \\ \text { MDAF + PI } & 0 & 2 \\ \text { LLH +MDAF + DDSP } & 0 & 1 \\ \text { LLH + VFC } & 0 & 2 \\ \text { LLH + VFC + PI } & 0 & 1 \\ \text { LLH + PI } & 0 & 1 \\ \text { LLH + VFC + MDAF } & 0 & 1\end{array}$

4

2

0

1

0

1

3

1

1

1

2

2

1

1

Table 2. This table shows the number of resting and overground endoscopic findings. Left laryngeal hemiplegia (LLH), Palatal Instability (PI), Dorsal Displacement of Soft Palate (DDSP), Medial Deviation of Aryepiglottic Fold (MDAF), Nasopharyngeal Collapse (NPC), Vocal Fold Collapse (VFC), Ventro-medial Luxation of the Apex of the Corniculate Process (VLAC)

In our study, PI (n:6) was the most occurred abnormality at resting endoscopy. However, it was observed that DDSP developed in two of these 6 horses in OE examination [Figure 3]. In addition, PI and LLH cases were diagnosed in 8 horses at resting endoscopy were as simple abnormalities. When these cases are evaluated via OE, it was observed MDAF, DDSP, PI, and VFC along with LLH in 6 of 8 horses. Also, while diagnosed by resting endoscopy in one of 8 horses has a grade III LLH, and another horse has with grade IV LLH resting asymmetry of the larynx, in OE examination of the same case observed LLH with VFC [Figure 4] and treatment options were submitted.

\section{Discussion}

The diagnosis of URT abnormalities in horses does not accurately achieved during an endoscopic examination at rest and therefore there is still the need to make a definitive diagnosis [8,30]. The overground endoscopic examination enables safely the diagnosis of dynamic URT abnormalities at exercise in natural conditions and the most suitable treatment options of dynamic URT pathology in racehorses [23]. The endoscopic examination during the exercise using Overground or Highspeed treadmill (HSTE) endoscopy has been used the diagnosis of URT abnormalities in all horses with a simple or complex abnormality, that the horses some complaints such as abnormal respiratory noises and fade abruptly are derived from the horse owners, trainers, and jockeys [18,19,31]. In our study, the diagnostic efficiency of OE and resting endoscopic examinations in horses with simple and complex URT abnormalities was evaluated comparatively and OE was found useful in the diagnosis of both simple and complex abnormality. 
Left laryngeal hemiplagea (LHH) is generally seen on the left side, which is a distal neuropathy of the recurrent laryngeal nerve Complete paralysis Grade IV occurred, which cannot change during exercise, with resting endoscopy but LLH rarely determine as a simple case, so that it can be diagnosed with exercising endoscopy, still plays a significant role in determining. During performing exercising endoscopy can be graded as either simple or complex case by enabling to gather knowledge regarding the VFC, which is commonly seen in horses with LLH and can be especially detected during exercise as a complex case [2].

Palatal dysfunction is closely associated with the syndromes of DDSP and PI [17]. Palatal instability (PI) may be a predecessor to DDSP $[8,19,27]$. It was suggested that significant variations in the results of endoscopy at rest and during exercise were identified, which indicate that resting endoscopy may not be sufficient to predict the occurrence of pathology during exercise [31]. Dynamic endoscopic examination either HSTE or overground must be required to make a correct diagnosis and to discuss the way of the treatment. We diagnosed two DDSP simple and one DDSP complex cases by using OE that referred as to PI while doing resting endoscopy. The most important part is here to decide the way of treatment, especially for complex cases.

The limiting point of this study has not been compared overground endoscopic findings with HSTE. There is absolutely a need to further conducted research on the validity of both HSTE and OE, how these two techniques compare with each other to determine the functional abnormalities. Because of this an ideal study would involve the comparison of treadmill endoscopy and overground endoscopy in the same horses and compared their findings as a resting one. However, performing exercising endoscopy either overground or high speed treadmill the occurrence between the two testing conditions were no differences to diagnose VFC and MDAF, the retrospective studies have been reported that DDSP was seen more frequently with HSTE than with overground endoscopy, because horses examined during HSTE under more strenuous condition [15,28], which occurs artificial condition to provide strenuous that has longer distance with increasing the inclines, while at lower speed when compare the overground one.

In contrast to the treadmill test, this study was showed the average speed of the exercise test could more readily be compared with the speed during the race the overground tests were performed the last meters at a fast gallop, where horses showed the peak performance at the begging of fatigue symptoms, which differs for the usual overground test and naturally it gives more strenuous condition. OE technique is more closely comparable to race conditions than HSTE in which the endpoint of the exercise test is typically the point when an abnormality is detected [18].

Trainers preferred short inclined gallops; it was often necessary to perform the overground exercise test in intervals rather than as a continuous test. Interval exercise depended on the horse's performance and the usual rider can easily understand the behavior of the horse under normal conditions and ridden until fatigue able to give rest periods to recovery [29]. The continuous test may cause some injuries for this reason [19].

In this study abnormal noise and fade abruptly occurred during the last meters of the race, according to this situation we preferred the overground endoscope because it's cheaper and safer than HSTE and not require an additional person. It is necessary at least three experience people to use HSTE and the horse must train how to run on the treadmill before the examination. OE examination on a polytrack racecourse is the nearest of a race experience.

The disadvantage of OE equipment using in this study was the heavyweight $(14 \mathrm{~kg})$ of the saddle, which existed the monitor and battery left and right side of it. Also recording unit and the camera of endoscope were unable to controlled remotely in case of any screen loose or mucus accumulation, and these problems have been eliminated in different and latest models of the same company.

This study highlights the importance of usage overground endoscopy to detect the multiple forms of dynamic functional abnormalities of URT, which can be missed with resting one, and it plays an important role in choosing the straightway of the treatment. 


\section{Conclusions}

Even a very small functional change in the URT can lead to poor performance in racehorses which occurs under strenuous exercise. The overground endoscopic examination has a useful diagnostic value in determining the cause of abnormal respiratory noise and/or fade abruptly which is made during routine training. Presented study, an overground endoscopic examination was found to be important because this technique gives us a way to find complex dynamic obstructions of the upper respiratory tract and helps us to decide on treatment way. The results of this study give an important message to veterinary practitioners: if you can't diagnose the problem accurately then the treatment might not be efficient.

Funding: This research received no external funding.

Author Contributions: All authors have read and agreed to the published version of the manuscript.

Conflicts of Interest: The authors declare no conflict of interest.

\section{References}

1. Franklin, SH. Dynamic collapse of the upper respiratory tract: a review. Equine Vet. Educ 2008, 20, $212-224$.

2. Barakzai, SZ. Respiratory endoscopy. 1st ed. London: Elsevier, 2007.

3. McCann, J. Differential diagnosis of abnormal respiratory noises in the exercising horse. In Pract 2000, 22, 370-81.

4. Rakestraw, P.C.; Hackett, R.P.; Duchrame, N.G.; Nielan, G.J.; Erb H.N. Arytenoid cartilage movement in resting and exercising horse. Vet.Surg.1991, 20, 122-127.

5. Holcombe, S.J.; Ducharme, N.G. Abnormalities of the upper airway. In Equine sports Medicine and surgery. Hinchcliff, KW., Kaneps, AJ., Geor, RJ; Saunders: Philadelphia, USA, 2004. pp. 559-98.

6. Barakzai, S.Z.; Dixon, P.M. Correlation of resting and exercising endoscopic findings for horses with dynamic laryngeal collapse and palatal dysfunction. Equine Vet J 2011, 43, 18-23.

7. Go, L.; Barton, A.K.; Ohnesorge, B.; Evaluation of laryngeal function under the influence of various head and neck positions during exercise in 58 performance horses. Equine Vet Edu 2014, 26, 41-7.

8. Kannegieter, N.J.; Dore, M.L., Endoscopy of the upper respiratory tract during treadmill exercise: A clinical study of 100 horses. Aust Vet J 1995, 72, 101- 107.

9. Morris, E.A.; Seeherman, H.J. Evaluation of upper respiratory tract function during strenuous exercise in racehorses. $J$ Am Vet Med Assoc, 1990, 196, 431-438.

10. Lane, J.G. Recurrent laryngeal neuropathy. Australian Equine Veterinary Association 1993, 15, 173- 192.

11. Boles, C.L.; Raker, C.W.; Wheat, J.F. Epiglottic entrapment by arytenoepiglottic folds in the horse. J.Am.vet.med. Ass 1978, 172, 338-342.

12. Tamzali, Y.;Serraud, N; Baup, B.; Desmaizieres, L.M. How to perform endsocopy during exercise without a treadmill.Proc.Am. Ass.Equine Practnrs 2008, 54,24-28.

13. Franklin, S.H.; Naylor, J.R.; Burn, J.F.; Morgan, J.; Allen, K.J. Preliminary clinical trials of an ambulatory telemetric endoscopy system for the assessment of the upper respiratory tract in the exercising horse. In: Proceedings of the British Equine Veterinary Association, Newmarket, UK, 2008; Equine Veterinary Journal.

14. Desmaizieres, L.M., Serraud, N., Plainfosse, B., Michel, A., Tamzali, Y. Dynamic respiratory endoscopy without treadmill in 68 performance Standardbred, Thoroughbred and saddle horses under natural training conditions. Equine Vet.J. 2009, 41, 347-352.

15. Allen, K.J.; Franklin, S.H. Comparisons of overground endoscopy and treadmill endoscopy in UK Thoroughbred racehorses. Equine Vet.J. 2010, 42, 186-191.

16. Martin, B.B.; Reef, V.B.; Parente, E.J.; Sage, A.D. Causes of poor performance of horses during training, racing, or showing in 348 cases. J. Am. vet. med. Ass. 2000, 216, 554-558.

17. Dart, A.J.; Dowling, B.A.; Hodgson, D.R.; Rose, R.J. Evaluation of highspeed treadmill videoendoscopy for diagnosis of upper respiratory tract dysfunction in horses. Aust. vet. J 2001, 79, 109-112.

18. Tan, R.H.; Dowling, B.A.; Dart, A.J. High-speed treadmill videoendoscopic examination of the upper respiratory tract in the horse: the results of 291 clinical cases. Vet. J. 2005, 170, 243-248.

19. Lane, J.G.; Bladon, B.; Little, D.R.; Naylor, J.R.; Franklin, S.H. Dynamic obstructions of the equine upper respiratory tract. Part 2: comparison of endoscopic findings at rest and during high-speed treadmill exercise of 600 Thoroughbred racehorses. Equine vet. J. 2006, 38, 401-407.

20. Lane, J.G.; Bladon, B.; Little, D.R.; Naylor, J.R.; Franklin, S.H. Dynamic obstructions of the equine upper respiratory tract. Part 1: comparison of endoscopic findings at rest and during high-speed treadmill exercise of 600 Thoroughbred racehorses. Equine vet. J. 2006, 38, 393-399. 
21. Evans, D. Exercise testing in the field. In Equine Sports Medicine and Surgery, Hinchcliff, KW., Kaneps, AJ., Geor, RJ; Saunders: Philadelphia, USA, 2004. pp. 19-31.

22. Pollock P.J.; Reardon R.J.; Parkin T.D. Dynamic respiratory endoscopy in 67 thoroughbred racehorses training under normal ridden exercise conditions. Equine Vet J 2009, 41(4), 354-60.

23. Kumas, C.; Maden, M. Evaluation of the Dynamic (Overground) Endoscopy Procedure in the Diagnosis of Upper Respiratory Tract Diseases Affecting Performance of Racehorses. Kafkas University Faculty of Vet.Med. J. 2013, 19 (supplement-A), 55-60.

24. King, D.S.;Tulleners, E.; Martin B.B.; Parente E.J.; Boston, R. Clinical experiences with axial deviation of the aryepiglottic folds in 52 racehorses. Vet.Surg. 2001, 30, 151-160.

25. Dart, A.J.; Dowling, B.A.; Smith, C.L. Upper airway dysfunction associated with collapse of the apex of the corniculate process of the left arytenoid cartilage during exercise in 15 horses. Vet.Surg.2005, 34, 543-547.

26. Boyle, A.G.; Martin,B.B.; Jr, Davidson, E.J.; Durando, M.M.; Birks, E.K. Dynamic pharyngeal collapse in racehorses. Equine vet. J. 2006, Suppl, 546-550.

27. Robertson J.T. Dorsal displacement of the soft palate. In Current Techniques in Equine Surgery and Lameness, 2nd ed.; White, N.A., Moore, J.N., Saunders: Philadelphia, USA, 1998. Pp.131-135.

28. Van Erck-Wetergen, E.; Frippiart, T.; Dupius, M.C.; Richard, E.; Art,T.; Desmaizieres, L.M. Upper airway endoscopy: are track and treadmill operations comparable? In: Proceedings of the World Equine Airways Society Symposium, 2009. pp. 254-255.

29. Midgley, A.W.; McNaughton, L.R.; Carroll, S.; Physiological determinants of time to exhaustion during intermittent treadmill running at vVO2max. Int. J. sports. Med. 2007, 28, 273-280.

30. Parente, E.J.; Martin, B.B. Correlation between standing endoscopic examinations and those made during high-speed exercise in horses - 150 cases. Proceedings of Am. Assoc. Equine Practnrs, 1995, 41, 170.

31. Kelly, P.G.; Reardon, R.J.M.; Johnston, M.S.; Pollock, P.J. Comparison of dynamic and resting endoscopy of the upper portion of the respiratory tract in 57 Thoroughbred yearlings. Equine Veterinary Journal, 2013, 45, 700-704. 